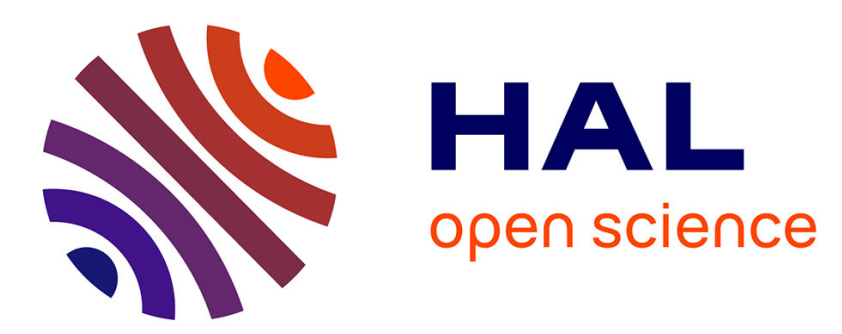

\title{
Sufficient Conditions for a Central Limit Theorem to Assess the Error of Randomized Quasi-Monte Carlo Methods
}

\author{
Marvin K Nakayama, Bruno Tuffin
}

\section{- To cite this version:}

Marvin K Nakayama, Bruno Tuffin. Sufficient Conditions for a Central Limit Theorem to Assess the Error of Randomized Quasi-Monte Carlo Methods. 2021 - Winter Simulation Conference, Dec 2021, Phoenix, United States. pp.1-12. hal-03196351

\section{HAL Id: hal-03196351 \\ https://hal.inria.fr/hal-03196351}

Submitted on 12 Apr 2021

HAL is a multi-disciplinary open access archive for the deposit and dissemination of scientific research documents, whether they are published or not. The documents may come from teaching and research institutions in France or abroad, or from public or private research centers.
L'archive ouverte pluridisciplinaire HAL, est destinée au dépôt et à la diffusion de documents scientifiques de niveau recherche, publiés ou non, émanant des établissements d'enseignement et de recherche français ou étrangers, des laboratoires publics ou privés. 


\title{
Sufficient Conditions for a Central Limit Theorem to Assess the Error of Randomized Quasi-Monte Carlo Methods
}

\author{
Marvin K. Nakayama \\ Computer Science Department \\ New Jersey Institute of Technology \\ Newark, NJ 07102, USA
}

\author{
Bruno Tuffin \\ Inria, Univ Rennes, CNRS, IRISA \\ Campus de Beaulieu, 263 Avenue Général Leclerc \\ 35042 Rennes, FRANCE
}

\begin{abstract}
Randomized quasi-Monte Carlo (RQMC) can produce an estimator of a mean (i.e., integral) with rootmean-square error that shrinks at a faster rate than Monte Carlo's. While RQMC is often employed to provide a confidence interval (CI) for the mean, this approach implicitly assumes that the RQMC estimator obeys a central limit theorem (CLT), which has not been established for most RQMC settings. To address this, we provide various conditions that ensure an RQMC CLT, as well as an asymptotically valid CI, and examine the tradeoffs in our restrictions. Our sufficient conditions, depending on the regularity of the integrand, generally require that the number of randomizations grows sufficiently fast relative to the number of points used from the low-discrepancy sequence.
\end{abstract}

\section{INTRODUCTION}

Analyzing a stochastic model frequently involves computing the mean performance $\mu$. Often, $\mu$ can be expressed as an integral of a function $h$ over an $s$-dimensional unit hypercube $[0,1]^{s}$ for some fixed $s \geq 1$. Such integrals for $s>1$ are typically analytically intractable, leading to the use of numerical methods, including simulation. As these techniques incur error, we should give a measure of the error.

Monte Carlo (MC) estimates $\mu$ via random sampling (Asmussen and Glynn 2007). Repeatedly feeding independent and identically distributed (i.i.d.) uniformly distributed random vectors on $[0,1]^{s}$ into integrand $h$ produces i.i.d. outputs, which are averaged to yield the MC estimator. The method affords simple error estimation through a confidence interval (CI). Based on a central limit theorem (CLT), a CI uses the sample variance to provide a computable (probabilistic) measure of the MC error. But as the sample size $n$ (i.e., number of evaluations of $h$ ) grows, the CI and the MC estimator's root-mean-square error (RMSE) shrink at a slow rate $n^{-1 / 2}$; adding another digit of precision requires a 100 -fold increase in $n$.

To obtain a more efficient estimator, quasi-Monte Carlo (QMC) replaces the i.i.d. uniforms driving the MC method with $n$ deterministic points from a low-discrepancy sequence (e.g., a lattice or digital net), designed to more evenly fill $[0,1]^{s}$ than a typical random sample; see Niederreiter (1992) and Lemieux (2009). When the integrand $h$ has bounded Hardy-Krause variation, the Koksma-Hlawka inequality (e.g., Section 2.2 of Niederreiter 1992) shows that the QMC error decreases as $O\left(n^{-1}(\ln n)^{s}\right)$ as $n \rightarrow \infty$, better than the rate at which MC's RMSE shrinks. While theoretically useful, the Koksma-Hlawka inequality has limited practical value as its bound is not easily computed and is often quite loose.

Randomized QMC (RQMC) suggests a way to obtain a computable error bound: randomize the QMC points $r \geq 2$ i.i.d. times and build a CI from the sample variance of the resulting $r$ i.i.d. estimators; e.g., see Tuffin (2004), Section 6.2 of Lemieux (2009), and L'Ecuyer (2018). For a given (large) computation budget of $n$ integrand evaluations, we specify the number $m$ of points used from each randomized sequence so that $m r \approx n$. To choose such an allocation $(m, r)$, a common rule of thumb recommends taking $r$ small (e.g., $10 \leq r \leq 30$ ) so that $m$ is correspondingly large to benefit from QMC's superior convergence rate. 


\section{Nakayama and Tuffin}

The RQMC CI's validity implicitly assumes that the RQMC estimator obeys a Gaussian CLT. When $m \rightarrow \infty$ and $r$ is fixed, Loh (2003) establishes a CLT that covers only a computationally prohibitive form of RQMC, limiting its practical use. But more generally, a Gaussian limit is not guaranteed; e.g., randomly shifting a lattice leads to non-normal limits (as $m \rightarrow \infty$ for fixed $r$ ) (L'Ecuyer et al. 2010). Thus, while intuitively appealing, the CI lacks rigorous theoretical justification for most RQMC methods.

Our paper addresses these shortcomings. We provide sufficient conditions on both $h$ and $(m, r)$ that ensure the RQMC estimator obeys a CLT, as well as an asymptotically valid CI (AVCI). We focus on the setting where both $m, r \rightarrow \infty$ since a Gaussian limit may not hold for fixed $r$. We will show tradeoffs in our restrictions on $h$ and $(m, r)$ : more stringent limitations on $h$ lead to looser constraints on $(m, r)$. But in all cases, the RQMC RMSE shrinks faster than for the corresponding MC estimator with sample size $n=m r$.

The rest of the paper unfolds as follows. Section 2 builds our study's basic framework. We present general conditions that yield a CLT and AVCI in Sections 3 and 4, respectively. Section 5 provides simpler sufficient conditions for a CLT or AVCI, and gives graphical comparisons of the alternative restrictions. Concluding remarks are in Section 6. All formal proofs appear in Nakayama and Tuffin (2021).

\section{NOTATION AND FRAMEWORK}

For an integrand $h:[0,1]^{s} \rightarrow \Re$ on the unit hypercube of fixed dimension $s \geq 1$, the goal is to compute

$$
\mu=\int_{[0,1]^{s}} h(u) \mathrm{d} u=\mathbb{E}[h(U)]
$$

where random vector $U \sim \mathscr{U}[0,1]^{s}$ with $\mathscr{U}[0,1]^{s}$ denoting a uniform distribution on $[0,1]^{s}, \sim$ means "is distributed as", and $\mathbb{E}$ represents the expectation operator. We can think of $h$ as a (complicated) simulation program that transforms $s$ i.i.d. 1-dimensional uniform random numbers into observations from specified input distributions, which are then used to produce an output of the random performance of a stochastic system, so $\mu$ is its mean. We next explain how to apply MC, QMC, and RQMC to estimate $\mu$.

\subsection{Monte Carlo}

With MC, we generate $n$ i.i.d. copies $U_{1}, U_{2}, \ldots, U_{n}$ of $U \sim \mathscr{U}[0,1]^{s}$, and compute $\widehat{\mu}_{n}^{\mathrm{MC}}=\sum_{i=1}^{n} h\left(U_{i}\right) / n$ as the MC estimator of $\mu$. Let $\psi^{2} \equiv \operatorname{Var}[h(U)]$, with $\operatorname{Var}[\cdot]$ the variance operator, and assume that $0<\psi^{2}<\infty$. The MC estimator is unbiased (i.e., $\mathbb{E}\left[\widehat{\mu}_{n}^{\mathrm{MC}}\right]=\mu$ ), as are all the estimators of $\mu$ that we consider, so

$$
\operatorname{RMSE}\left[\widehat{\mu}_{n}^{\mathrm{MC}}\right]=\frac{\psi}{\sqrt{n}} .
$$

The MC estimator obeys a Gaussian CLT $\sqrt{n}\left[\widehat{\mu}_{n}^{\mathrm{MC}}-\mu\right] / \psi \Rightarrow \mathscr{N}(0,1)$ as $n \rightarrow \infty$ (Billingsley 1995, Theorem 27.1), where $\Rightarrow$ denotes convergence in distribution, and $\mathscr{N}\left(a, b^{2}\right)$ is a normal random variable with mean $a$ and variance $b^{2}$. Let $\widehat{\psi}_{n}^{2}=\sum_{i=1}\left[h\left(U_{i}\right)-\widehat{\mu}_{n}^{\mathrm{MC}}\right]^{2} /(n-1)$ be the sample variance of the i.i.d. $h\left(U_{i}\right)$. For a desired confidence level $0<\gamma<1$, we can exploit the CLT to construct an approximate $\gamma$-level CI for $\mu$ as $I_{n, \gamma}^{\mathrm{MC}} \equiv\left[\widehat{\mu}_{n}^{\mathrm{MC}} \pm z_{\gamma} \widehat{\psi}_{n} / \sqrt{n}\right]$, where the critical point $z_{\gamma}$ satisfies $\Phi\left(z_{\gamma}\right)=1-(1-\gamma) / 2$ and $\Phi$ is the $\mathscr{N}(0,1)$ cumulative distribution function (CDF). Providing a probabilistic measure of the MC estimator's error, $I_{n, \gamma}^{\mathrm{MC}}$ is an AVCI in the sense that $\lim _{n \rightarrow \infty} P\left(\mu \in I_{n, \gamma}^{\mathrm{MC}}\right)=\gamma$ (Asmussen and Glynn 2007, p. 71).

\subsection{Quasi-Monte Carlo}

QMC replaces MC's i.i.d. uniforms with carefully placed deterministic points from a low-discrepancy sequence $\Xi=\left(\xi_{i}\right)_{i \geq 1}$, such as a digital net (e.g., a Sobol' sequence) or lattice; see, e.g., Chapters 3-5 of Niederreiter (1992). Using the first $n$ points from $\Xi$ leads to QMC approximating $\mu$ by $\widehat{\mu}_{n}^{\mathrm{Q}}=\sum_{i=1}^{n} h\left(\xi_{i}\right) / n$. We can bound the error $\left|\widehat{\mu}_{n}^{\mathrm{Q}}-\mu\right|$ via the Koksma-Hlawka inequality (Niederreiter 1992, Section 2.2):

$$
\left|\widehat{\mu}_{n}^{\mathrm{Q}}-\mu\right| \leq V_{\mathrm{HK}}(h) D_{n}^{*}(\Xi)
$$




\section{Nakayama and Tuffin}

for all $n>1$, where $D_{n}^{*}(\Xi)$ is the star-discrepancy of the first $n$ points in $\Xi$, and $V_{\mathrm{HK}}(h)$ is the Hardy-Krause variation of the integrand $h$. In (2), $V_{\mathrm{HK}}(h) \geq 0$ quantifies the "roughness" of $h$, and $D_{n}^{*}(\Xi) \in[0,1]$ measures the "nonuniformity" of $\Xi$. Low-discrepancy sequences often have

$$
D_{n}^{*}(\Xi)=O\left(n^{-1}(\ln n)^{s}\right), \quad \text { as } n \rightarrow \infty,
$$

where $f(n)=O(g(n))$ (resp., $f(n)=\Theta(g(n)))$ as $n \rightarrow \infty$ for functions $f$ and $g$ means that there exist positive constants $a_{0}, a_{1}$, and $n_{0}$ such that $|f(n)| \leq a_{1}|g(n)|$ (resp., $a_{0}|g(n)| \leq|f(n)| \leq a_{1}|g(n)|$ ) for all $n \geq n_{0}$. Thus, if $V_{\mathrm{HK}}(h)<\infty$, (2) and (3) imply that the QMC error shrinks as $O\left(n^{-1}(\ln n)^{s}\right)$ as $n \rightarrow \infty$, better than the $\Theta\left(n^{-1 / 2}\right)$ rate at which MC's RMSE decreases. While theoretically useful, the bound in (2) has limited practical value as it is not easily computed and is often quite loose. There are other related error bounds (e.g., Hickernell 1998; Hickernell 2018; Lemieux 2006; Niederreiter 1992), but all suffer from the same issues.

\subsection{Randomized Quasi-Monte Carlo}

RQMC applies i.i.d. randomizations of the QMC sequence $\Xi$ to produce i.i.d. estimators of $\mu$, and builds an approximate CI via their sample variance. A randomization creates from $\Xi$ another sequence $\Xi^{\prime} \equiv\left(U_{i}^{\prime}\right)_{i \geq 1}$ that retains the low-discrepancy properties of $\Xi$. Each $U_{i}^{\prime} \sim \mathscr{U}[0,1]^{s}$, but the points in $\Xi^{\prime}$ are dependent. RQMC employs such a randomization $r \geq 1$ i.i.d. times, and for each $j=1, \ldots, r$, let $\Xi_{j}^{\prime} \equiv\left(U_{i, j}^{\prime}\right)_{i \geq 1}$ be the $j$ th randomized sequence. Given a computation budget of $n$ integrand evaluations, we specify the number $m$ of points to use from each $\Xi_{j}^{\prime}$ so that $m r \approx n$, leading to the RQMC estimator of $\mu$ as

$$
\widehat{\mu}_{m, r}^{\mathrm{RQ}}=\frac{1}{r} \sum_{j=1}^{r} X_{j}, \quad \text { where } \quad X_{j}=\frac{1}{m} \sum_{i=1}^{m} h\left(U_{i, j}^{\prime}\right) .
$$

The $X_{j}, j=1, \ldots, r$, are i.i.d., and let $\widehat{\sigma}_{m, r}^{2}=\sum_{j=1}^{r}\left(X_{j}-\widehat{\mu}_{m, r}^{\mathrm{RQ}}\right)^{2} /(r-1)$ be their sample variance when $r \geq 2$. We then arrive at a possible $\gamma$-level CI $I_{m, r, \gamma}^{\mathrm{RQ}} \equiv\left[\widehat{\mu}_{m, r}^{\mathrm{RQ}} \pm z_{\gamma} \widehat{\sigma}_{m, r}\right]$ for $\mu$.

The literature includes several methods to construct $\Xi^{\prime}$, including scrambled digital nets (Owen 1995; Owen 1997) and digital shifts (L'Ecuyer 2018). To simplify the discussion, we describe only one approach: random shifts (Cranley and Patterson 1976). Here, randomization $j$ generates a single $U_{j} \sim \mathscr{U}[0,1]^{s}$ and adds it (modulo 1) to each point in $\Xi$, so the $i$ th point in the $j$ th randomized sequence $\Xi_{j}^{\prime}$ is $U_{i, j}^{\prime}=\left\langle U_{j}+\xi_{i}\right\rangle$, where $\langle x\rangle$ is the modulo- 1 operator applied to each coordinate of $x \in \mathfrak{R}^{s}$. The $U_{j}$ across randomizations $j=1,2, \ldots, r$, are independent. It is easy to show that each $U_{i, j}^{\prime} \sim \mathscr{U}[0,1]^{s}$, so $\widehat{\mu}_{m, r}^{\mathrm{RQ}}$ and each $X_{j}$ are unbiased estimators of $\mu$. But for each randomization $j$, the sequence $\Xi_{j}^{\prime}$ has dependent points because they all share the same uniform $U_{j}$.

With random shifts, each randomized sequence $\Xi_{j}^{\prime}$ satisfies (Tuffin 1997, Theorem 2)

$$
D_{m}^{*}\left(\Xi_{j}^{\prime}\right) \leq 4^{s} D_{m}^{*}(\Xi) \text {. }
$$

Thus, if $V_{\mathrm{HK}}(h)<\infty$, the estimator $X_{j}$ in (4) from a single randomization of $m$ points satisfies $\mathrm{RMSE}\left[X_{j}\right]=$ $O\left(m^{-1}(\log m)^{s}\right)$ as $m \rightarrow \infty$, an improvement over the $\Theta\left(m^{-1 / 2}\right)$ rate in (1) for MC using the same number $m$ of integrand evaluations. Even faster convergence rates can be achieved for special classes of functions and specific sequences $\Xi$ called lattice rules (Tuffin 1998; L'Ecuyer and Lemieux 2000).

Although intuitively appealing, the $\mathrm{CI} I_{m, r, \gamma}^{\mathrm{RQ}}$ in general lacks theoretical justification, as it implicitly relies on $\widehat{\mu}_{m, r}^{\mathrm{RQ}}$ obeying a Gaussian CLT. For $m \rightarrow \infty$ with $r \geq 1$ fixed, Loh (2003) establishes an RQMC CLT that covers solely the case of fully nested scrambling of a digital net, which is computationally prohibitive, limiting its adoption by practitioners. For random shifts of a lattice, the RQMC estimator $\widehat{\mu}_{m, r}^{\mathrm{RQ}}$ may not obey a Gaussian CLT as $m \rightarrow \infty$ for fixed $r \geq 1$, as shown by L'Ecuyer et al. (2010). Indeed, they prove that for $r=1$, the limiting error distribution has simple non-Gaussian forms for dimension $s=1$, and $s>1$ generally leads to non-Gaussian limits with no such easy characterizations, so the same holds for any fixed $r \geq 1$. Thus, we see the need for general Gaussian CLTs for RQMC, which is our aim. 


\section{Nakayama and Tuffin}

\subsection{Assumptions and Preliminary Results}

We want to study the asymptotic behavior of the RQMC estimator in (4) as the computation budget $n$ for the number of integrand evaluations grows large. To do this, we take the number $m \equiv m_{n} \geq 1$ of points from the randomized sequence and the number $r \equiv r_{n} \geq 1$ of randomizations to be functions of $n$ satisfying Assumption 1.A $m_{n} r_{n} \leq n$ for each $n \geq 1$, with $m_{n} \rightarrow \infty, r_{n} \rightarrow \infty$, and $m_{n} r_{n} / n \rightarrow 1$ as $n \rightarrow \infty$.

Under Assumption 1.A, the RQMC estimator in (4) becomes

$$
\widehat{\mu}_{m_{n}, r_{n}}^{\mathrm{RQ}}=\frac{1}{r_{n}} \sum_{j=1}^{r_{n}} X_{n, j}, \quad \text { where } \quad X_{n, j}=\frac{1}{m_{n}} \sum_{i=1}^{m_{n}} h\left(U_{i, j}^{\prime}\right),
$$

so $X_{n, j}$ averages $h$ on the first $m_{n}$ points of the $j$ th randomized sequence. Our goal is to provide conditions on $h$ and $\left(m_{n}, r_{n}\right)$ that yield (as $\left.n \rightarrow \infty\right)$ a Gaussian CLT (Section 3) or AVCI (Section 4). Other papers (e.g., Glynn 1987; Damerdji 1994) adopt frameworks akin to Assumption 1.A to study MC methods for analyzing steady-state behavior via multiple replications or batching.

Assumption 1.A requires $r_{n} \rightarrow \infty$ because otherwise, the limiting error distribution may not be Gaussian, as noted at the end of Section 2.3. We simplify the discussion by further having $m_{n} \rightarrow \infty$ in Assumption 1.A, but this is not necessary; Nakayama and Tuffin (2021) also analyze the special case that $m_{n} \equiv m_{0}$ for a fixed $m_{0} \geq 1$. Section 5 will adopt the following specialization of Assumption 1.A.

Assumption 1.B $m_{n}=n^{c}$ and $r_{n}=n^{1-c}$ with $c \in(0,1)$.

We should define, e.g., $m_{n}=\left\lfloor n^{c}\right\rfloor$ and $r_{n}=\left\lfloor n^{1-c}\right\rfloor\left(\lfloor\cdot\rfloor\right.$ is the floor function) so that $m_{n}$ and $r_{n}$ are integers, but for simplicity, we ignore this technicality. Section 5 will determine constraints on $h$ and $c \in(0,1)$ that secure a CLT or AVCI, and in each case, the optimal such $c$ that minimizes the rate at which $\operatorname{RMSE}\left[\widehat{\mu}_{m_{n}, r_{n}}^{\mathrm{RQ}}\right]$ shrinks as $n \rightarrow \infty$. Also, we will examine the tradeoffs in the conditions on $h$ and $c$.

For a randomized sequence $\Xi^{\prime}$ constructed from scrambling or a digital shift of a digital net, or for a randomly shifted lattice rule, the randomization preserves the partitioning structure of the original sequence $\Xi$ : a randomly shifted lattice is still a lattice, and scrambling or digitally shifting a digital net retains the original sequence's finer-grain properties (Owen 1995; Owen 1997; L'Ecuyer 2018). Moreover, these $\Xi^{\prime}$ obey similar discrepancy bounds as $\Xi$. Specifically, consider any low-discrepancy sequence $\Xi$ for which (3) holds, so there exists some constant $0<w_{0}<\infty$ such that $D_{m}^{*}(\Xi) \leq w_{0} m^{-1}(\ln m)^{s}$ for all $m>1$. Then its random shift $\Xi^{\prime}$ satisfies $D_{m}^{*}\left(\Xi^{\prime}\right) \leq w_{0}^{\prime} m^{-1}(\ln m)^{s}$ with $w_{0}^{\prime}=4^{s} w_{0}$ by (5), and scrambling or digital shifting digital nets yields analogous bounds. Thus, all of these randomizations fulfill the following assumption, which we use to analyze RQMC estimators when $V_{\mathrm{HK}}(h)<\infty$.

Assumption 2 For the RQMC method used, there exists a constant $0<w_{0}^{\prime}<\infty$ such that each randomized sequence $\Xi^{\prime}$ satisfies $D_{m}^{*}\left(\Xi^{\prime}\right) \leq w_{0}^{\prime} m^{-1}(\ln m)^{s}$ for all $m>1$, where $w_{0}^{\prime}$ depends on the RQMC method but not on the randomization's realization (e.g., of $U \sim \mathscr{U}[0,1]^{s}$ in a random shift).

We often will further impose one of the following conditions on the integrand $h$. The conditions are presented in order of decreasing strength (see Proposition 1 below), and Section 5 will show that this leads to corresponding tradeoffs in our conditions on $\left(m_{n}, r_{n}\right)$ to ensure a CLT or AVCI.

Assumption 3.A The integrand $h$ is of bounded Hardy-Krause variation, i.e., $V_{\mathrm{HK}}(h)<\infty$.

Assumption 3.B The integrand $h$ is bounded; i.e., $|h(u)| \leq t_{0}$ for all $u \in[0,1]^{s}$ for some constant $t_{0}<\infty$. Assumption 3.C There exists $b>0$ such that $\mathbb{E}\left[|h(U)-\mu|^{2+b}\right]<\infty$, where $U \sim \mathscr{U}[0,1]^{s}$.

Limiting the roughness of $h$ over $[0,1]^{s}$, Assumption 3.A imposes substantial restrictions; it does not hold, e.g., in dimension $s \geq 2$ when $h$ is an indicator function (so $\mu$ is a probability) with discontinuities not lining up with the coordinate axes (Owen and Rudolf 2020). In contrast, Assumption 3.C constrains the heaviness of the tails of the distribution of $h(U)$.

Proposition 1 Assumption 3.A is strictly stronger than Assumption 3.B, itself strictly stronger than Assumption 3.C. 


\section{Nakayama and Tuffin}

Using different conditions on $h$, we next derive two bounds on absolute central moments of the estimator $X_{n, 1}$ in (6) from a single randomization. The first lemma, for $V_{\mathrm{HK}}(h)<\infty$ (Assumption 3.A), follows from Theorem 2 of Tuffin (1997); the second applies Minkowski's inequality (Billingsley 1995, eq. (5.40)) when Assumption 3.C holds for $2+b$ replaced by $q \geq 1$.

Lemma 1 Under Assumptions 1.A, 2, and 3.A, for any $q>0$ and for all $n$ such that $m_{n}>1$,

$$
\eta_{n, q} \equiv \mathbb{E}\left[\left|X_{n, 1}-\mu\right|^{q}\right] \leq \mathbb{E}\left[\left(V_{\mathrm{HK}}(h) D_{m_{n}}^{*}\left(\Xi^{\prime}\right)\right)^{q}\right] \leq\left(\frac{w_{0}^{\prime} V_{\mathrm{HK}}(h)\left(\ln m_{n}\right)^{s}}{m_{n}}\right)^{q}<\infty
$$

Lemma 2 Under Assumption 1.A, for any $q \geq 1$, if $\mathbb{E}\left[|h(U)-\mu|^{q}\right]<\infty$ for $U \sim \mathscr{U}[0,1]^{s}$, then $\eta_{n, q} \leq$ $\mathbb{E}\left[|h(U)-\mu|^{q}\right]$ for every $n$.

For a single randomization of $m$ points, RQMC typically has $\sigma_{m} \equiv\left(\operatorname{Var}\left[\sum_{i=1}^{m} h\left(U_{i, 1}^{\prime}\right) / m\right]\right)^{1 / 2}=O\left(m^{-\alpha}\right)$ as $m \rightarrow \infty$ with $\alpha>1 / 2$ (e.g., see (7) when $V_{\mathrm{HK}}(h)<\infty$ ). This improves on MC's RMSE convergence rate, which satisfies RMSE $\left[\widehat{\mu}_{m}^{\mathrm{MC}}\right]=\sqrt{\operatorname{Var}\left[\widehat{\mu}_{m}^{\mathrm{MC}}\right]}=\psi m^{-1 / 2}$ by (1). Assume the following limit exists:

$$
\alpha_{*}=-\lim _{m \rightarrow \infty} \frac{\ln \left(\sigma_{m}\right)}{\ln (m)}
$$

so $\alpha_{*}$ is the constant such that $\sigma_{m}$ decreases, as $m \rightarrow \infty$, at a rate (ignoring leading coefficients and lower-order terms) strictly faster than $m^{-\alpha_{*}+\varepsilon}$ and strictly slower than $m^{-\alpha_{*}-\varepsilon}$ for every $\varepsilon>0$; i.e., $\sigma_{m}=o\left(m^{-\alpha_{*}+\varepsilon}\right)$ and $\sigma_{m}=\omega\left(m^{-\alpha_{*}-\varepsilon}\right)$ as $m \rightarrow \infty$ for any $\varepsilon>0$, where $f(m)=o(g(m))$ as $m \rightarrow \infty$ means that $f(m) / g(m) \rightarrow 0$ as $m \rightarrow \infty$, and $f(m)=\omega(g(m))$ as $m \rightarrow \infty$ means that $f(m) / g(m) \rightarrow \infty$ as $n \rightarrow \infty$. By (7),

$$
\alpha_{*} \geq 1 \quad \text { when } V_{\mathrm{HK}}(h)<\infty,
$$

as in Assumption 3.A, and more generally, as is typical of RQMC, we assume that

$$
\alpha_{*}>\frac{1}{2}
$$

The value of $\alpha_{*}$ depends on the particular integrand $h$ and the RQMC method applied, but not on how $\left(m_{n}, r_{n}\right)$ or $c$ are specified in Assumptions 1.A and 1.B.

For any randomized sequence $\Xi^{\prime}=\left(U_{i}^{\prime}\right)_{i \geq 1}$, let $E_{m}\left(\Xi^{\prime}\right)=\frac{1}{m} \sum_{i=1}^{m} h\left(U_{i}^{\prime}\right)-\mu$ be the error of the estimator based on the first $m$ points. Then define its exponential rate as

$$
\alpha\left(\Xi^{\prime}\right)=-\lim _{m \rightarrow \infty} \frac{\ln \left(\left|E_{m}\left(\Xi^{\prime}\right)\right|\right)}{\ln (m)}
$$

assuming the limit always exists, and define the worst-case rate among all randomizations as

$$
\alpha^{\prime}=\inf _{\Xi^{\prime}} \alpha\left(\Xi^{\prime}\right)
$$

Thus, for any randomized sequence $\Xi^{\prime}$, we see that $\left|E_{m}\left(\Xi^{\prime}\right)\right|=o\left(m^{-\alpha+\varepsilon}\right)$ and $\left|E_{m}\left(\Xi^{\prime}\right)\right|=\omega\left(m^{-\alpha-\varepsilon}\right)$ as $m \rightarrow \infty$ for each $\varepsilon>0$, and $\alpha^{\prime} \leq \alpha_{*}$ always holds.

Assumption 4 The convergence rate exponent $\alpha^{\prime}$ of the worst-case error among all randomizations is the same as the standard deviation rate exponent $\alpha_{*}$; i.e., $\alpha^{\prime}=\alpha_{*}$.

Corollary 1 in Section 5.1 will later show that when Assumption 4 holds, the RQMC estimator $\widehat{\mu}_{m_{n}, r_{n}}^{\mathrm{RQ}}$ will obey a CLT for $\left(m_{n}, r_{n}\right)=\left(n^{c}, n^{1-c}\right)$ for any $c \in(0,1)$ (Assumption 1.B). However, establishing Assumption 4 in practice may be difficult, so much of our paper focuses on providing other more verifiable sufficient conditions that secure a CLT. 


\section{Nakayama and Tuffin}

For a given total number $n$ of integrand evaluations, RQMC papers often suggest choosing $m_{n}$ as large as possible to gain from the fast convergence rate of QMC. But as noted at the end of Section 2.3, we still should specify $r_{n}$ big enough to ensure a Gaussian CLT. Under Assumptions 1.B, 2 and 3.A, (7) implies

$$
\operatorname{RMSE}\left[\widehat{\mu}_{m_{n}, r_{n}}^{\mathrm{RQ}}\right] \leq \frac{\left[w_{0}^{\prime} V_{\mathrm{HK}}(h)\left(\ln m_{n}\right)^{s} / m_{n}\right]}{\sqrt{r_{n}}}=\Theta\left(\frac{(c \ln n)^{s}}{n^{(1+c) / 2}}\right),
$$

so larger $c$ leads to faster convergence. While $c=1$ minimizes the RMSE bound, a Gaussian CLT may not be guaranteed, as noted earlier at the end of Section 2.3.

\section{GENERAL CONDITIONS FOR A CENTRAL LIMIT THEOREM}

We next study limiting properties of $\widehat{\mu}_{m_{n}}^{\mathrm{RQ}} r_{n}$ in (6) as $n \rightarrow \infty$. The estimator averages $X_{n, 1}, X_{n, 2}, \ldots, X_{n, r_{n}}$, but their distribution changes with $n$, complicating the asymptotic analysis. To develop a theoretical framework for handling this under Assumption 1.A, note that $\left(X_{n, j}\right)_{n=1,2, \ldots ; j=1,2, \ldots, r_{n}}$ forms a triangular array (Billingsley 1995, p. 359), also called a double array. In a triangular array, the $r_{n}$ variables within a row $n$ are independent, but there may be dependence across rows. While the general formulation allows for the CDFs of the $r_{n}$ variables within a row $n$ to differ, RQMC actually has

$$
X_{n, 1}, X_{n, 2}, \ldots, X_{n, r_{n}} \text { are i.i.d., each with some distribution } F_{n},
$$

where $F_{n}$ may change with $n$, as occurs in (6). To preclude trivialities, we impose another assumption, without which the exact result is eventually always returned by the RQMC estimator.

Assumption $5 \sigma_{m_{n}}^{2} \equiv \operatorname{Var}\left[X_{n, 1}\right]>0$ for all sufficiently large $n$.

The Lindeberg and Lyapounov CLTs (Billingsley 1995, Theorems 27.2 and 27.3) apply for the RQMC structure in (13). To set them up, write the variance of the sum of the $r_{n}$ random variables in (13) as $s_{n}^{2} \equiv r_{n} \sigma_{m_{n}}^{2}$. Denote the CDF of $X_{n, j}-\mu$ by $G_{n}$, which does not depend on $j$ by (13). Note that $\sigma_{m_{n}}^{2}=\int_{y \in \Re} y^{2} \mathrm{~d} G_{n}(y)$, and let $\tau_{n}^{2}(t)=\int_{|y|>t s_{n}} y^{2} \mathrm{~d} G_{n}(y)$ for $t>0$. Then the RQMC estimator in (6) satisfies the following.

Theorem 1 If Assumptions 1.A and 5 hold and also the Lindeberg condition

$$
\frac{\tau_{n}^{2}(t)}{\sigma_{m_{n}}^{2}} \rightarrow 0, \quad \text { as } n \rightarrow \infty, \quad \forall t>0,
$$

then the RQMC estimator in (6) satisfies the CLT

$$
\frac{\widehat{\mu}_{m_{n}, r_{n}}^{\mathrm{RQ}}-\mu}{\sigma_{m_{n}} / \sqrt{r_{n}}} \Rightarrow \mathscr{N}(0,1), \quad \text { as } n \rightarrow \infty .
$$

Also, (14) holds if, for some $b>0, \mathbb{E}\left[\left|X_{n, 1}-\mu\right|^{2+b}\right]<\infty$ for each $n$ and the Lyapounov condition holds:

$$
\frac{\mathbb{E}\left[\left|X_{n, 1}-\mu\right|^{2+b}\right]}{r_{n}^{b / 2} \sigma_{m_{n}}^{2+b}} \rightarrow 0, \quad \text { as } n \rightarrow \infty .
$$

The Lindeberg condition (14) constrains the tail behavior of $G_{n}$. In the general setting of independent but not identically distributed $X_{n, j}, 1 \leq j \leq r_{n}$, the analogous version of (14) (Billingsley 1995, eq. (27.8)) ensures that the contribution of any single $X_{n, j}$ to their sum's variance $s_{n}^{2}$ is negligible for large $n$. We can show (Billingsley 1995, p. 361) that (14) is even necessary for the CLT (15) when (13) holds. Imposing restrictions on moments rather than tail properties, the Lyapounov condition (16) can sometimes be easier to apply than the Lindeberg condition (14). Section 5.1 will obtain sufficient conditions for (14) or (16) under Assumption 1.B to secure Theorem 1 for each of our Assumptions 3.A-3.C on the integrand $h$. 


\section{Nakayama and Tuffin}

\section{ASYMPTOTICALLY VALID CONFIDENCE INTERVAL}

To build a CI from the CLT (15), suppose that $r_{n} \geq 2$, which Assumption 1.A ensures for all $n$ large enough. As $X_{n, j}, j=1,2, \ldots, r_{n}$, are i.i.d. by (13), their sample variance $\widehat{\sigma}_{m_{n}, r_{n}}^{2}=\sum_{j=1}^{r_{n}}\left(X_{n, j}-\widehat{\mu}_{m_{n}, r_{n}}^{\mathrm{RQ}}\right)^{2} /\left(r_{n}-1\right)$ provides an unbiased estimator of $\sigma_{m_{n}}^{2}=\operatorname{Var}\left[X_{n, 1}\right]$. For a given desired confidence level $\gamma \in(0,1)$, we get

$$
I_{m_{n}, r_{n}, \gamma}^{\mathrm{RQ}} \equiv\left[\widehat{\mu}_{m_{n}, r_{n}}^{\mathrm{RQ}} \pm z_{\gamma} \widehat{\sigma}_{m_{n}, r_{n}} / \sqrt{r_{n}}\right]
$$

as the RQMC CI for $\mu$. The next result imposes conditions guaranteeing that $I_{m_{n}, r_{n}, \gamma}^{\mathrm{RQ}}$ is an AVCI in the sense that (20) below holds.

Theorem 2 Suppose that Assumptions 1.A and 5 hold. Also, suppose that $\mathbb{E}\left[\left(X_{n, 1}-\mu\right)^{4}\right]<\infty$ and that

$$
\frac{\mathbb{E}\left[\left(X_{n, 1}-\mu\right)^{4}\right]}{r_{n} \sigma_{m_{n}}^{4}} \rightarrow 0, \quad \text { as } n \rightarrow \infty .
$$

Then

$$
\frac{\widehat{\mu}_{m_{n}, r_{n}}^{\mathrm{RQ}}-\mu}{\widehat{\sigma}_{m_{n}, r_{n}} / \sqrt{r_{n}}} \Rightarrow \mathscr{N}(0,1), \quad \text { as } n \rightarrow \infty,
$$

and

$$
\lim _{n \rightarrow \infty} P\left(\mu \in I_{m_{n}, r_{n}, \gamma}^{\mathrm{RQ}}\right)=\gamma .
$$

As (18) is the same as (16) for $b=2$, Theorem 1 implies CLT (15), which is expressed in terms of the exact $\sigma_{m_{n}}$. But the left side of (19) instead uses the estimator $\widehat{\sigma}_{m_{n}, r_{n}}$. Theorem 2's conditions further ensure $\widehat{\sigma}_{m_{n}, r_{n}} / \sigma_{m_{n}} \Rightarrow 1$ as $n \rightarrow \infty$, so Slutsky's theorem (Billingsley 1995, p. 340) verifies (19), securing AVCI (20). Section 5.1 will provide sufficient conditions under Assumption 1.B that yield Theorem 2 for two of our conditions on the integrand $h$ (Assumptions 3.A and 3.C).

\section{ANALYSIS WHEN $\left(m_{n}, r_{n}\right)=\left(n^{c}, n^{1-c}\right)$ (ASSUMPTION 1.B)}

Assumption 1.B specializes Assumption 1.A by taking $\left(m_{n}, r_{n}\right)=\left(n^{c}, n^{1-c}\right)$ for some $c \in(0,1)$. We next will determine the values of $c$ that imply CLT (15) through Theorem 1 or that guarantee AVCI (20) via Theorem 2. For those $c$, we then find the ones leading to RMSE $\left[\widehat{\mu}_{m_{n}, r_{n}}^{\mathrm{RQ}}\right]$ shrinking fastest as $n \rightarrow \infty$.

Under Assumption 1.B, we have that $m_{n}=n^{c}$ with $c \in(0,1)$, so (8) implies that

$$
\sigma_{m_{n}}=\omega\left(n^{-c \alpha_{*}-\varepsilon}\right) \quad \text { and } \quad \sigma_{m_{n}}=o\left(n^{-c \alpha_{*}+\varepsilon}\right) \quad \text { as } n \rightarrow \infty \text {, for all } \varepsilon>0 .
$$

Taking $\varepsilon>0$ arbitrarily small in (21) leads to $\sigma_{m_{n}} \approx \Theta\left(n^{-c \alpha_{*}}\right)$ as $n \rightarrow \infty$. Thus, a combination of $r_{n}=n^{1-c}$ with (6) and (13) yields, for any $c \in(0,1)$,

$$
\operatorname{RMSE}\left[\widehat{\mu}_{m_{n}, r_{n}}^{\mathrm{RQ}}\right]=\frac{\sigma_{m_{n}}}{\sqrt{r_{n}}} \approx \Theta\left(n^{-v\left(\alpha_{*}, c\right)}\right) \quad \text { as } n \rightarrow \infty, \quad \text { where } v\left(\alpha_{*}, c\right) \equiv c\left[\alpha_{*}-\frac{1}{2}\right]+\frac{1}{2} .
$$

Our assumption (10) guarantees that $v\left(\alpha_{*}, c\right)>1 / 2$ for $c \in(0,1)$. Hence, the convergence rate of RQMC's RMSE for any $c$ in Assumption 1.B is better than $\operatorname{RMSE}\left[\widehat{\mu}_{n}^{\mathrm{MC}}\right]=\Theta\left(n^{-v_{\mathrm{MC}}}\right)$ as $n \rightarrow \infty$ for MC, where

$$
v_{\mathrm{MC}} \equiv \frac{1}{2}
$$

by (1). For any fixed $\alpha_{*}$ satisfying (10), $v\left(\alpha_{*}, c\right)$ strictly increases in $c$ by (22), so RQMC's RMSE shrinks faster for larger $c$. We thus want to determine how large $c$ can be and still ensure CLT (15) or AVCI (20). 


\section{Nakayama and Tuffin}

Section 5.1 will provide various corollaries of the CLT and AVCI theorem in Sections 3 and 4. Each such Corollary $k$ will result in restricting $c$ as

$$
c<c_{k}\left(\alpha_{*}\right)
$$

for some $0<c_{k}\left(\alpha_{*}\right) \leq 1$ depending on the particular Corollary $k$. As we will see, most of the $c_{k}\left(\alpha_{*}\right)$ are strictly decreasing in $\alpha_{*}$. Thus, as $\alpha_{*}$ increases, (24) further restricts the choices of $c$, thereby reducing the maximum allowable number of QMC points and increasing the minimum number of randomizations because $\left(m_{n}, r_{n}\right)=\left(n^{c}, n^{1-c}\right)$. But by (8), larger $\alpha_{*}$ corresponds to a better convergence rate for the estimator based on a single randomization, so in some sense, securing a CLT or AVCI often entails hampering better QMC performance.

Because (22) implies that larger $c$ leads to RMSE shrinking at a faster rate, the "optimal" $c$ that maximizes the rate subject to the constraint (24) is $c=c_{k}\left(\alpha_{*}\right)-\delta$ for infinitesimally small $\delta>0$. Accordingly, an analysis akin to the arguments applied to achieve (22) arrives at the optimal approximate rate:

$$
\operatorname{RMSE}\left[\widehat{\mu}_{m_{n}, r_{n}}^{\mathrm{RQ}}\right] \approx \Theta\left(n^{-v_{k}\left(\alpha_{*}\right)}\right) \quad \text { as } n \rightarrow \infty,
$$

where, for each Corollary $k$ (and $k^{\prime}$ ) in Section 5.1, the exponent $v_{k}\left(\alpha_{*}\right)$ appears below in (26).

Proposition 2 Under Assumption 1.B and (10), the optimal approximate RMSE rate exponent in (25) is

$$
v_{k}\left(\alpha_{*}\right) \equiv c_{k}\left(\alpha_{*}\right)\left(\alpha_{*}-\frac{1}{2}\right)+\frac{1}{2}>v_{\mathrm{MC}}
$$

for $v_{\mathrm{MC}}$ in (23), so RQMC outdoes MC. If $c_{k}\left(\alpha_{*}\right)=1$ in (24), then $v_{k}\left(\alpha_{*}\right)=\alpha_{*}$. Also, for any $k$ and $k^{\prime}$,

$$
v_{k}\left(\alpha_{*}\right)>v_{k^{\prime}}\left(\alpha_{*}\right) \text { if and only if } c_{k}\left(\alpha_{*}\right)>c_{k^{\prime}}\left(\alpha_{*}\right) .
$$

When $c_{k}\left(\alpha_{*}\right)=1$, (24) becomes the weakest possible constraint satisfying Assumption 1.B. In this case, Proposition 2 implies that $v_{k}\left(\alpha_{*}\right)=\alpha_{*}$, so the RMSE of the multiple-randomization RQMC estimator $\widehat{\mu}_{m_{n}, r_{n}}^{\mathrm{RQ}}$ decreases at about the same rate as for a single randomization with full length $m=n$.

The next subsection will specialize $c_{k}\left(\alpha_{*}\right)$ in (24) and $v_{k}\left(\alpha_{*}\right)$ in (26) for various corollaries. Section 5.2 will compare the resulting values graphically.

\subsection{Corollaries of Theorems 1 and 2}

We first provide a corollary of Theorem 1 based on Assumption 4, which imposes constraints on both the integrand and RQMC sequence.

Corollary 1 Suppose that Assumptions $1 . \mathrm{B}, 4$, and 5 hold. If $c<1 \equiv c_{1}\left(\alpha_{*}\right)$, then the Lindeberg condition (14) and CLT (15) hold. Moreover, (25) and (26) have $v_{k}\left(\alpha_{*}\right)=v_{1}\left(\alpha_{*}\right) \equiv \alpha_{*}$.

Under Assumption 4 (the worst-case error and the standard deviation decrease at the same exponential rate), Corollary 1 secures a CLT for $\left(m_{n}, r_{n}\right)=\left(n^{1-\varepsilon}, n^{\varepsilon}\right)$ with $\varepsilon>0$ as small as we wish. Thus, although $r_{n} \rightarrow \infty$ is needed, choosing $\varepsilon>0$ small allows taking a large number $m_{n}=n^{1-\varepsilon}$ of points from the low-discrepancy sequence, which enables exploiting QMC's superior convergence rates.

As establishing Assumption 4 may be difficult in practice, we next provide other conditions that are more readily verifiable to ensure CLT (15). We give corollaries corresponding to each of our restrictions on the integrand $h$ in Assumptions 3.A-3.C, which are in decreasing order of strength (Proposition 1). We first specialize (16) of Theorem 1 to establish a CLT when $V_{\mathrm{HK}}(h)<\infty$, which enables using Lemma 1.

Corollary 2 Suppose that Assumptions 1.B, 2, 3.A $\left(V_{\mathrm{HK}}(h)<\infty\right)$, and 5 hold. Also, suppose that for some constant $\lambda \in(0,1)$,

$$
c<\frac{1-\lambda}{2 \alpha_{*}-1-\lambda} \equiv c_{2}\left(\alpha_{*}\right)
$$




\section{Nakayama and Tuffin}

where $\lambda$ may be chosen arbitrarily small. Then the Lyapounov condition (16) and CLT (15) hold. Moreover, for each $\alpha_{*} \geq 1$, as in (9), $c_{2}\left(\alpha_{*}\right)$ satisfies $0<c_{2}\left(\alpha_{*}\right) \leq 1$, and (25) and (26) have $v_{k}\left(\alpha_{*}\right)=v_{2}\left(\alpha_{*}\right)$ with

$$
v_{2}\left(\alpha_{*}\right) \equiv \frac{2 \alpha_{*}-1-\lambda \alpha_{*}}{2 \alpha_{*}-1-\lambda}, \quad \text { where } \quad \frac{1}{2}<v_{2}\left(\alpha_{*}\right) \leq 1 .
$$

Corollary 2 allows taking $\lambda \in(0,1)$ as arbitrarily small in (28) and (29), so $c_{2}\left(\alpha_{*}\right) \approx 1 /\left(2 \alpha_{*}-1\right)$ and $v_{2}\left(\alpha_{*}\right) \approx 1$. The next corollary of Theorem 1 exploits (14) to yield a CLT when the integrand $h$ is bounded.

Corollary 3 Suppose that Assumptions 1.B, 3.B ( $h$ is bounded), and 5 hold. If

$$
c<\frac{1}{2 \alpha_{*}+1} \equiv c_{3}\left(\alpha_{*}\right),
$$

then the Lindeberg condition (14) and CLT (15) hold. Moreover, for each $\alpha_{*}>1 / 2$, as in (10), $c_{3}\left(\alpha_{*}\right)$ satisfies $0<c_{3}\left(\alpha_{*}\right)<1 / 2$, and (25) and (26) have $v_{k}\left(\alpha_{*}\right)=v_{3}\left(\alpha_{*}\right)$ with

$$
v_{3}\left(\alpha_{*}\right) \equiv \frac{2 \alpha_{*}}{2 \alpha_{*}+1}, \quad \text { where } \quad \frac{1}{2}<v_{3}\left(\alpha_{*}\right)<1 .
$$

The following corollary of Theorem 1 imposes a moment condition on $h(U)$ (Assumption 3.C) to apply Lemma 2 to (16) to obtain a CLT, in contrast to requiring $V_{\mathrm{HK}}(h)<\infty$, as in Corollary 2.

Corollary 4 Suppose that Assumptions 1.B, 3.C (finite absolute central moment of order $2+b$ for some $b>0$ ), and 5 hold. If

$$
c<\frac{1}{2 \alpha_{*}\left(1+\frac{2}{b}\right)+1} \equiv c_{4}\left(\alpha_{*}, b\right),
$$

then the Lyapounov condition (16) and CLT (15) hold. Moreover, for each $b>0$ and $\alpha_{*}>1 / 2$, as in (10), $c_{4}\left(\alpha_{*}, b\right)$ satisfies $0<c_{4}\left(\alpha_{*}, b\right)<1 / 2$, and (25) and (26) have $v_{k}\left(\alpha_{*}\right)=v_{4}\left(\alpha_{*}, b\right)$ with

$$
v_{4}\left(\alpha_{*}, b\right) \equiv \frac{2 \alpha_{*}\left(1+\frac{1}{b}\right)}{2 \alpha_{*}\left(1+\frac{2}{b}\right)+1}, \quad \text { where } \quad \frac{1}{2}<v_{4}\left(\alpha_{*}, b\right)<1 \text {. }
$$

For $I_{m_{n}, r_{n}, \gamma}^{\mathrm{RQ}}$ in (17) to be AVCI (20), Theorem 2 assumes that (18) holds, which yields the CLTs in (15) and (19). We next consider conditions that enable verifying AVCI.

Corollary 5 Suppose that Assumptions 1.B, 2, 3.A $\left(V_{\mathrm{HK}}(h)<\infty\right)$, and 5 hold. If

$$
c<\frac{1}{4 \alpha_{*}-3} \equiv c_{5}\left(\alpha_{*}\right),
$$

then the CLT (19) and AVCI (20) hold. Moreover, for each $\alpha_{*} \geq 1$, as in (9), $c_{5}\left(\alpha_{*}\right)$ satisfies $0<c_{5}\left(\alpha_{*}\right) \leq 1$, and (25) and (26) have $v_{k}\left(\alpha_{*}\right)=v_{5}\left(\alpha_{*}\right)$ with

$$
v_{5}\left(\alpha_{*}\right) \equiv \frac{3 \alpha_{*}-2}{4 \alpha_{*}-3}, \quad \text { where } \quad \frac{3}{4}<v_{5}\left(\alpha_{*}\right) \leq 1 .
$$

While Corollary 5 requires $V_{\mathrm{HK}}(h)<\infty$, we next ensure AVCI instead through a moment condition.

Corollary 6 Suppose that Assumptions 1.B and 5 hold, as well as Assumption 3.C (finite absolute central moment of order $2+b$ ) for $b=2$. If

$$
c<\frac{1}{4 \alpha_{*}+1} \equiv c_{6}\left(\alpha_{*}\right),
$$

then the CLT (19) and AVCI (20) hold. Moreover, for each $\alpha_{*}>1 / 2$, as in (10), $c_{6}\left(\alpha_{*}\right)$ satisfies $0<c_{6}\left(\alpha_{*}\right)<1 / 2$, and (25) and (26) have $v_{k}\left(\alpha_{*}\right)=v_{6}\left(\alpha_{*}\right)$ with

$$
v_{6}\left(\alpha_{*}\right) \equiv c_{6}\left(\alpha_{*}\right)\left(\alpha_{*}-\frac{1}{2}\right)+\frac{1}{2}=\frac{3 \alpha_{*}}{4 \alpha_{*}+1}, \quad \text { where } \quad \frac{1}{2}<v_{6}\left(\alpha_{*}\right)<\frac{3}{4} .
$$


Nakayama and Tuffin
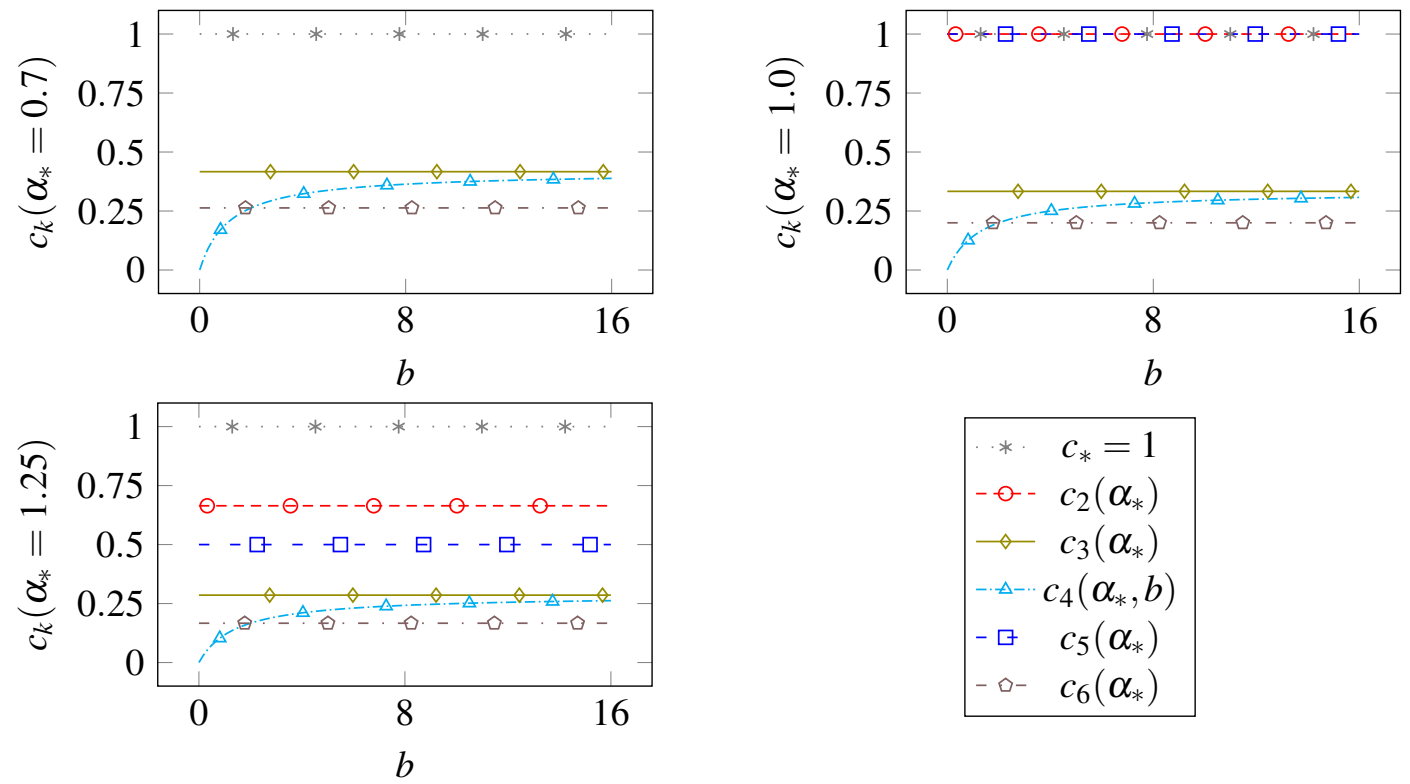

Figure 1: Plots of the upper bounds $c_{k}\left(\alpha_{*}\right)$ in (24) of $c$ in Assumption 1.B for different Corollaries $k$ from Section 5.1. The plots display the $c_{k}\left(\alpha_{*}\right)$ as functions of $b$ for different fixed values of $\alpha_{*}$. The upper left panel does not include $c_{2}\left(\alpha_{*}\right)$ and $c_{5}\left(\alpha_{*}\right)$ as these require $V_{\mathrm{HK}}(h)<\infty$, which then implies $\alpha_{*} \geq 1$ by (9). The plots show that stronger conditions on $h$ correspond to loosening constraints on $c$.

\subsection{Graphical Comparisons of the $c_{k}\left(\alpha_{*}\right)$ and the $v_{k}\left(\alpha_{*}\right)$}

For the Corollaries $k=2,3, \ldots, 6$ in Section 5.1, we next plot their upper bounds $c_{k}\left(\alpha_{*}\right)$ for $c$ as functions of $b$ (from Assumption 3.C) in Figure 1 for various fixed values of $\alpha_{*}>1 / 2$, as assumed in (10). (Our discussions omit $k=1$ as its Assumption 4 may be difficult to verify in practice; note nevertheless that $c_{1}\left(\alpha_{*}\right) \geq c_{k}\left(\alpha_{*}\right)$ and $v_{1}\left(\alpha_{*}\right)=\alpha_{*} \geq v_{k}\left(\alpha_{*}\right) \forall k \geq 2$.) Figure 2's left panel graphs the $c_{k}\left(\alpha_{*}\right)$ as functions of $\alpha_{*}$ instead, where larger $\alpha_{*}$ corresponds to better RQMC performance on a single randomization by (8), and the right panel does the same for the optimal approximate RMSE rate exponents $v_{k}\left(\alpha_{*}\right)$ of (25). The plots for Corollary $k=2$ set $\lambda=0.01$. The figures also show $c_{*}=1$ as Assumption 1.B requires $c \in(0,1)$. The right panel of Figure 2 further includes $v_{*}=1$ for reference.

Recall that Corollaries $k=2$ and 5 require Assumption 3.A $\left(V_{\mathrm{HK}}(h)<\infty\right), k=3$ imposes Assumption 3.B (bounded $h$ ), and $k=4$ and 6 employ Assumption 3.C (order- $(2+b)$ absolute central moment of $h(U)$ is finite). Proposition 1 gives a strict ordering of those assumptions' strengths. Figures 1 and 2 show the following properties, which Nakayama and Tuffin (2021) also establish analytically:

- $\quad c_{2}\left(\alpha_{*}\right)>c_{3}\left(\alpha_{*}\right)>c_{4}\left(\alpha_{*}, b\right)$ for each $b>0$ and $\alpha_{*}>1 / 2\left(c_{2}\left(\alpha_{*}\right)\right.$ being valid only when $\left.\alpha_{*} \geq 1\right)$, showing that stricter conditions on integrand $h$ permit larger values of $c$ ensuring CLT (15).

- $\quad c_{5}\left(\alpha_{*}\right)>c_{6}\left(\alpha_{*}\right)$ for each $b>0$ and $\alpha_{*}>1 / 2$, so a stronger condition on $h$ corresponds to a larger range of values of $c$ that yield AVCI (20).

- $c_{4}\left(\alpha_{*}, b\right)$ converges to $c_{3}\left(\alpha_{*}\right)$ as $b$ increases, which agrees with the principle that having a finite absolute central moment of order $2+b$ as $b \rightarrow \infty$ is "close" to meaning a bounded integrand. 
Nakayama and Tuffin
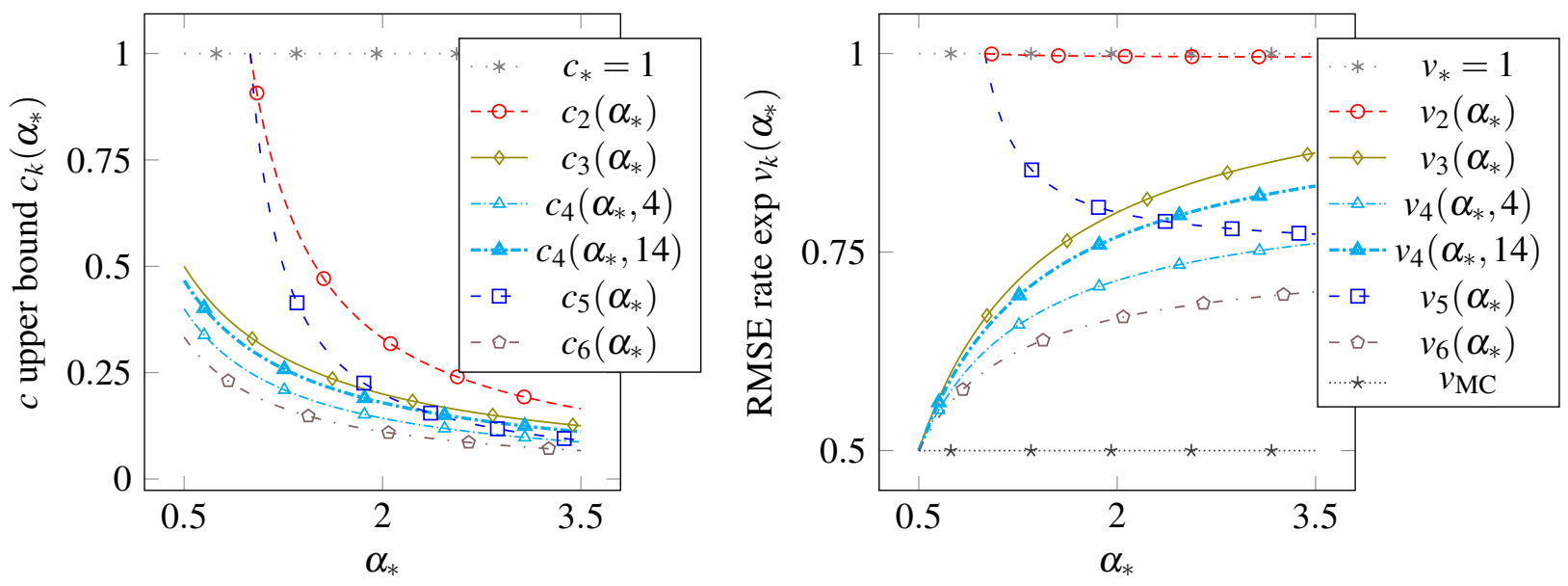

Figure 2: Plots of the upper bounds $c_{k}\left(\alpha_{*}\right)$ of $c$ (left panel) and the negative exponent $v_{k}\left(\alpha_{*}\right)$ of the optimal rate at which the estimator RMSE decreases (right panel) as functions of $\alpha_{*}$. Functions for $k=2$ and 5 require $V_{\mathrm{HK}}(h)<\infty$, so they are shown for only $\alpha_{*} \geq 1$ because of (9). Each $c_{k}\left(\alpha_{*}\right)$ decreases in $\alpha_{*}$, and most $v_{k}\left(\alpha_{*}\right)$ increase in $\alpha_{*}$, so better QMC behavior usually yields better RQMC performance.

- $\quad c_{4}\left(\alpha_{*}, b\right)$ grows as $b$ increases (i.e., more absolute central moments are finite), so additional effort can be put on the QMC part (i.e., $m_{n}=n^{c}$ can be larger) when using the moment conditions of Corollary 4 to establish a CLT.

- $\quad c_{5}\left(\alpha_{*}\right) \leq c_{2}\left(\alpha_{*}\right)$ and $c_{6}\left(\alpha_{*}\right)<c_{3}\left(\alpha_{*}\right)$, so securing AVCI (20) often (but not always) restricts $c$ more than what guarantees a CLT.

The $v_{k}\left(\alpha_{*}\right)$ share the same properties and orderings as the $c_{k}\left(\alpha_{*}\right)$ by (27).

In the left panel of Figure 2, the upper bounds $c_{k}\left(\alpha_{*}\right)$ on $c$ decrease as $\alpha_{*}$ grows, so ensuring CLT (15) or AVCI (20) for larger $\alpha_{*}$ requires putting more effort on the MC part (i.e., $r_{n}=n^{1-c}$ grows as $c$ decreases) and correspondingly less on the QMC (i.e., $m_{n}=n^{c}$ shrinks as $c$ gets smaller). By (26), the tradeoff could potentially harm the rate exponent $v_{k}\left(\alpha_{*}\right)$ governing how quickly the RQMC estimator's optimal RMSE decreases, but this does not occur for most $k$. The one exception is $v_{5}\left(\alpha_{*}\right)$ for the AVCI Corollary 5 when $V_{\mathrm{HK}}(h)<\infty$, which we explain by examining the corresponding $c_{5}\left(\alpha_{*}\right)$ in the left panel of Figure 2. While $c_{5}\left(\alpha_{*}\right)$ starts off at $\alpha_{*}=1$ very high, it quickly drops off, so $m_{n}$ must decrease rapidly as $\alpha_{*}$ grows to secure AVCI when $V_{\mathrm{HK}}(h)<\infty$, leading to less benefit from the QMC. Even so, we have that $v_{5}\left(\alpha_{*}\right)>v_{6}\left(\alpha_{*}\right)$ for all $\alpha_{*}$, so the optimal rate exponent when establishing AVCI is better for $V_{\mathrm{HK}}(h)<\infty$ than through the moment condition of Corollary 6.

\section{CONCLUDING REMARKS}

We presented conditions that ensure the RQMC estimator of a mean $\mu$ obeys a Gaussian CLT or guarantee AVCI. We also examined the tradeoffs in the restrictions. While our paper gave sufficient conditions, we are currently looking into relaxing the requirements. Other current work includes devising procedures to estimate the upper bounds $c_{k}\left(\alpha_{*}\right)$ in (24) and Section 5.1, which will allow practitioners to apply our theoretical results. We are further investigating analogous theory for biased estimators, as for quantiles.

\section{ACKNOWLEDGMENTS}

The authors would like to thank Art Owen for pointing out references, used in the proof of Proposition 1, showing that if the integrand $h$ has bounded Hardy-Krause variation, then $h$ is bounded. 


\section{Nakayama and Tuffin}

\section{REFERENCES}

Asmussen, S., and P. Glynn. 2007. Stochastic Simulation: Algorithms and Analysis. New York: Springer.

Billingsley, P. 1995. Probability and Measure. 3rd ed. New York: John Wiley and Sons.

Cranley, R., and T. N. L. Patterson. 1976. "Randomization of Number Theoretic Methods for Multiple Integration". SIAM Journal on Numerical Analysis 13(6):904-914.

Damerdji, H. 1994. "Strong Consistency of the Variance Estimator in Steady-State Simulation Output Analysis". Mathematics of Operations Research 19:494-512.

Glynn, P. W. 1987. "Limit Theorems for the Method of Replication". Stochastic Models 3(3):343-355.

Hickernell, F. J. 1998. “A Generalized Discrepancy and Quadrature Error Bound”. Mathematics of Computation 67(221):299-322.

Hickernell, F. J. 2018. "Error Analysis for Quasi-Monte Carlo Methods". In Monte Carlo and Quasi-Monte Carlo Methods: MCQMC 2016, edited by P. W. Glynn and A. B. Owen, 3-27. Berlin: Springer.

L'Ecuyer, P. 2018. "Randomized Quasi-Monte Carlo: An Introduction for Practitioners". In Monte Carlo and Quasi-Monte Carlo Methods: MCQMC 2016, edited by P. W. Glynn and A. B. Owen, 29-52. Berlin: Springer.

L'Ecuyer, P., and C. Lemieux. 2000. "Variance Reduction via Lattice Rules". Management Science 46(9):1214-1235.

L'Ecuyer, P., D. Munger, and B. Tuffin. 2010. "On the Distribution of Integration Error by Randomly-Shifted Lattice Rules". Electronic Journal of Statistics 4:950-993.

Lemieux, C. 2006. "Quasi-Random Number Techniques”. In Simulation, edited by S. G. Henderson and B. L. Nelson, Handbooks in Operations Research and Management Science, 351-380. Amsterdam, The Netherlands: Elsevier. Chapter 12.

Lemieux, C. 2009. Monte Carlo and Quasi-Monte Carlo Sampling. Series in Statistics. New York: Springer.

Loh, W.-L. 2003. "On the Asymptotic Distribution of Scrambled Net Quadrature”. Annals of Statistics 31(4):1282-1324.

Nakayama, M. K., and B. Tuffin. 2021. "Sufficient Conditions for Central Limit Theorems and Confidence Intervals for Randomized Quasi-Monte Carlo Methods". techreport hal-03196085, INRIA. https://hal.inria.fr/hal-03196085.

Niederreiter, H. 1992. Random Number Generation and Quasi-Monte Carlo Methods, Volume 63. Philadelphia: SIAM.

Owen, A. B. 1995. "Randomly Permuted (t,m,s)-Nets and (t,s)-Sequences". In Monte Carlo and Quasi-Monte Carlo Methods in Scientific Computing: Lecture Notes in Statistics, Volume 106, 299-317. Springer.

Owen, A. B. 1997. "Monte Carlo Variance of Scrambled Net Quadrature". SIAM Journal of Numerical Analysis 34:1884-1910.

Owen, A. B., and D. Rudolf. 2020. "A Strong Law of Large Numbers for Scrambled Net Integration". Technical report. https://arxiv.org/abs/2002.07859.

Tuffin, B. 1997. "Variance Reduction Applied to Product-Form Multi-Class Queuing Networks". ACM Transactions on Modeling and Computer Simulation 7(4):478-500.

Tuffin, B. 1998. "Variance Reduction Order Using Good Lattice Points in Monte Carlo Methods". Computing 61(4):371-378.

Tuffin, B. 2004. "Randomization of Quasi-Monte Carlo Methods for Error Estimation: Survey and Normal Approximation". Monte Carlo Methods and Applications 10(3-4):617-628.

\section{AUTHOR BIOGRAPHIES}

MARVIN K. NAKAYAMA is a professor in the Department of Computer Science at the New Jersey Institute of Technology. He received an M.S. and Ph.D. in operations research from Stanford University and a B.A. in mathematics-computer science from U.C. San Diego. He is a recipient of a CAREER Award from the National Science Foundation, and a paper he co-authored received the Best Theoretical Paper Award for the 2014 Winter Simulation Conference. He is an associate editor for ACM Transactions on Modeling and Computer Simulation, and served as the simulation area editor for the INFORMS Journal on Computing from 2007-2016. His research interests include simulation, modeling, statistics, risk analysis, and energy. His email address is marvin@njit.edu.

BRUNO TUFFIN received his PhD degree in applied mathematics from the University of Rennes 1 (France) in 1997. Since then, he has been with Inria in Rennes. His research interests include developing Monte Carlo and quasi-Monte Carlo simulation techniques for the performance evaluation of telecommunication systems and telecommunication-related economical models. He is currently Area Editor for INFORMS Journal on Computing and Associate Editor for ACM Transactions on Modeling and Computer Simulation. He has written or co-written three books (two devoted to simulation): Rare event simulation using Monte Carlo methods published by John Wiley \& Sons in 2009, La simulation de Monte Carlo (in French), published by Hermes Editions in 2010, and Telecommunication Network Economics: From Theory to Applications, published by Cambridge University Press in 2014. His email address is bruno.tuffin@inria.fr. 